\title{
Educación secundaria centrada en la persona
}

\section{Person-centered secondary education}

\section{Palabras clave}

Educación secundaria, inclusión, planificación centrada en la persona, profesionales.

\section{Keywords}

Secondary education, inclusion, person-centered planning, professionals.
"Porque todos miremos a través de gafas de cristales capacitantes"

1. Consideraciones generales sobre educación inclusiva

Siguiendo a Echeita et al. (20I7), entendemos que la educación inclusiva parte de un principio de no segregación del alumnado con dificultades y supone un desvelo por que todos los alumnos y alumnas estén en los centros escolares ordinarios y que alcancen los mayores logros en el desarrollo de sus capacidades al máximo nivel que les sea posible, garantizando su participación en términos de bienestar emocional. Por lo tanto, en el momento en el que no se garantice a cualquier estudiante alguno de estos tres aspectos (presencia, aprendizaje y participación), se le está colocando en una situación de vulnerabilidad.

Por ende, según el Marco de Acción (Unesco, I994) las escuelas inclusivas son aquellas que reconocen las diferentes necesidades de su alumnado y responden a ellas, adaptándose a los diferentes estilos y ritmos
Ana Benítez Jaén <ambenjae@upo.es>

Universidad Pablo de Olavide
Para citar:

Benítez, A. (2017): "Educación secundaria centrada en la persona". Revista Española de Discapacidad, 5 (2): I87-I93.

Doi: <https://doi.org/I0.5569/23405104.05.02.IO> 
de aprendizaje, asegurando una enseñanza de calidad a través de estudios apropiados, una buena organización escolar, una eficaz utilización de los recursos y una estrecha relación con sus comunidades.

La necesidad de garantizar a todos los estudiantes la participación y el aprendizaje escolar de calidad es hoy en día uno de los mayores desafíos para los sistemas educativos. Distintos estudios realizados en España en los últimos tiempos nos permiten afirmar que es la discapacidad intelectual, y particularmente en la etapa de Educación Secundaria Obligatoria, uno de los ámbitos donde se advierten con mayor intensidad las resistencias hacia la inclusión.

El informe Objetivos educativos europeos y españoles. Estrategia Educación Formación 2020 (2013) del Ministerio de Educación, Cultura y Deporte, afirma que las personas que abandonan los estudios se caracterizan por tener empleos más precarios, participan menos en el mercado de trabajo, tienen una menor probabilidad de empleo, están más expuestos a la temporalidad y obtienen menores salarios, reflejo de su menor productividad.

Muchas leyes educativas como la Ley General de Educación de España (hoy LOMCE) que en su artículo I, apartados b y e señalan:

"El sistema educativo español, configurado de acuerdo con los valores de la Constitución y asentado en el respeto a los derechos y libertades reconocidos en ella, se inspira en los siguientes principios:

b. La equidad, que garantice la igualdad de oportunidades (...).

e. La flexibilidad para adecuar la educación a la diversidad de aptitudes, intereses, expectativas y necesidades del alumnado, así como a los cambios que experimentan el alumnado y la sociedad".

Y en su artículo 2 señala, como primer objetivo, que: "El sistema educativo español se orientará a la consecución del pleno desarrollo de la personalidad y de las capacidades de los alumnos (...)".

Todos estos textos legales reconocen lo obvio, la existencia de necesidades educativas específicas asociadas a condiciones personales de capacidad intelectual, entre otras. Un sistema educativo debe ser aquel que promueve la personalización educativa y la flexibilidad curricular, permitiendo que cada alumno pueda llegar tan lejos como su capacidad y motivación permitan, a tanta velocidad como sus condiciones personales para aprender faciliten, con tanta amplitud como su curiosidad intelectual y creatividad favorezcan.

\section{La educación secundaria desde la educación inclusiva}

Giráldez et al. (2017), señalan los elementos favorecedores de la inclusión.

Desde nuestra perspectiva hemos ampliado el número de elementos favorecedores de la educación inclusiva. Consideramos que debe existir una planificación centrada en la persona y por extensión un currículum accesible y ajustado a las necesidades, características y capacidades del alumnado, rompiendo con la idea de que todos los niños tienen que aprender lo mismo a través del mismo material o actividad, en el mismo tiempo, puesto que los procesos de preparación para la inclusión sociolaboral son particulares y únicos para cada persona, por lo que es absolutamente necesario que cada joven participe plenamente en las acciones de toma de decisiones que se van dando. Además de una relación estrecha entre el currículum y unas habilidades y destrezas para una correctas inclusión sociolaboral.

En este sentido de preparación sociolaboral, la escuela secundaria juega un papel prioritario y fundamental tanto como espacio formativo de preparación para la futura inclusión como para ejercer la coordinación de los apoyos que deben permitir a los jóvenes con diversidad funcional 


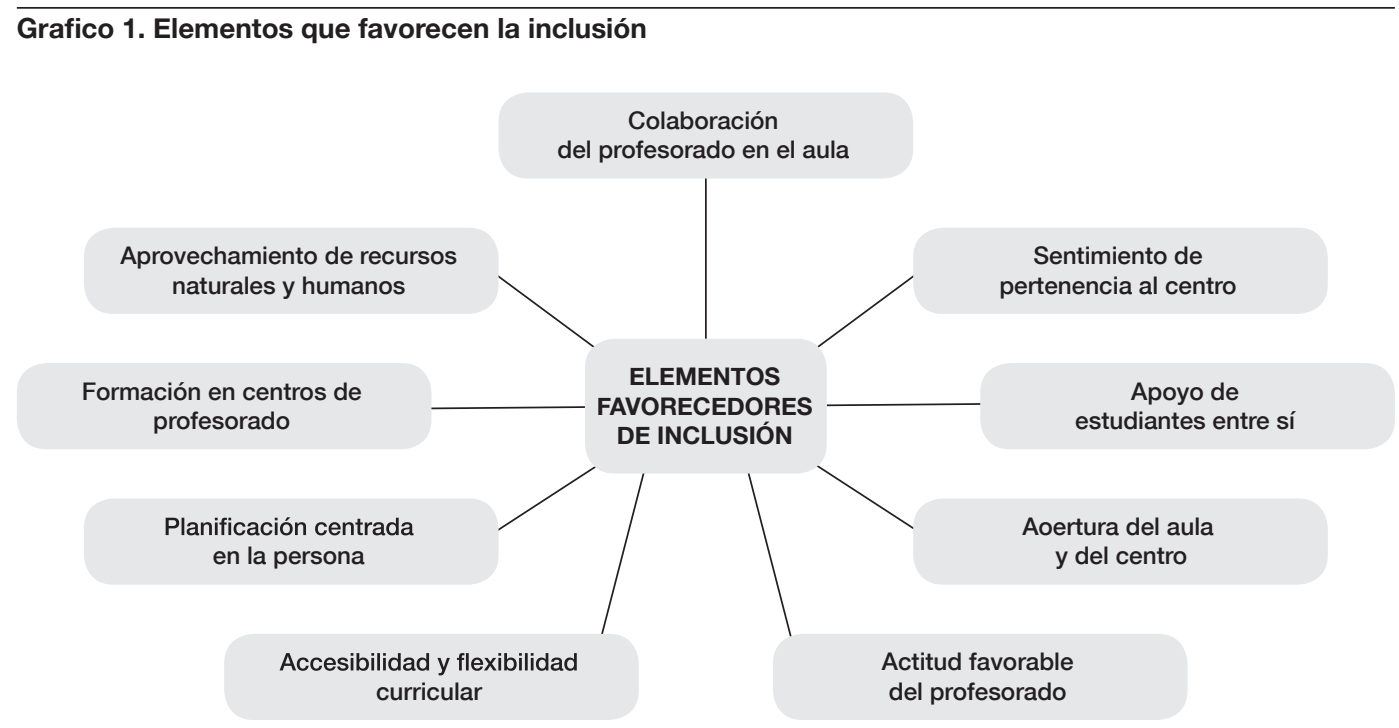

Fuente: elaboración propia, basado en Giráldez et al. (2017).

construir sus itinerarios personales más allá de la escuela.

En la línea, existe un amplio número de investigaciones que contribuyen con sus aportaciones a la concreción de los elementos clave que convierten a los centros de secundaria en factor decisivo para la inclusión laboral.

\subsection{Competencias docentes del profesorado de educación secundaria inclusiva}

Un estudio en la Comunidad de Madrid, el cual proponía evaluar las políticas de integración, encontró que aunque existe una mayoría de actores educativos favorables a la integración de alumnos considerados con "necesidades educativas especiales", un alto porcentaje de los profesores de enseñanza secundaria que trabajan con estudiantes con discapacidad intelectual disentían de esta posición (Marchesi et al. 2005).

De modo similar, otro estudio también de nivel nacional ha evaluado el avance de la inclusión en España. Dicho trabajo, que recoge la voz de expertos del movimiento asociativo de la discapacidad, e indirectamente de familias cuyos hijos se escolarizan en el sistema ordinario, concluye que la satisfacción de estos profesionales decrece a medida que se avanza hacia la enseñanza secundaria. Pero, además, la percepción de las organizaciones de personas con discapacidad intelectual tiende a ser más negativa que la del resto, cuando se evalúa específicamente lo que acontece en la etapa de enseñanza secundaria (Echeita et al., 2008).

Fernández Batanero (20I3), en la misma línea, nos presenta un estudio sobre las competencias docentes que propician buenas prácticas educativas en relación con la inclusión, desde la perspectiva del profesorado. El autor señala un conjunto de siete capacidades docentes, las cuales engloba bajo las siguientes competencias:

- Pedagógico-didácticas.

- De liderazgo.

- De gestión de grupo y aprendizaje cooperativo.

- De investigación.

- Interactivas.

- Éticas.

- Competencias sociales. 
En el siguiente cuadro, recogemos las competencias desglosadas en algunos de los indicadores de ejecución que indica el autor:

\begin{tabular}{|c|c|}
\hline \multicolumn{2}{|c|}{$\begin{array}{l}\text { Cuadro 1. Matriz de competencias del docente de educación secundaria en relación con la inclusión } \\
\text { educativa }\end{array}$} \\
\hline Competencias & Indicadores de ejecución \\
\hline Pedagógico-didácticas & $\begin{array}{l}\text { - Evalúa de forma continua para reorientar y cambiar estrategias. } \\
\text { - } \text { - Identifica y desarrolla estrategias innovadoras para favorecer la } \\
\text { inclusión de los alumnos con necesidades educativas. } \\
\text { - Acomoda la enseñanza y adecua los materiales. } \\
\text { - Crea situaciones diversas de enseñanza-aprendizaje. }\end{array}$ \\
\hline De liderazgo & $\begin{array}{l}\text { - Posee habilidades interpersonales. } \\
\text { - } \text { - Manea un clima de confianza y comunicación. } \\
\text { - Actúa de nexo entre las diferentes diversidades del centro educativo. }\end{array}$ \\
\hline $\begin{array}{l}\text { De gestión de grupo y } \\
\text { aprendizaje cooperativo }\end{array}$ & $\begin{array}{l}\text { - Gestiona las metodologías de trabajo didáctico y las tareas de } \\
\text { aprendizaje. } \\
\text { - Propicia diálogos y situaciones destinadas a la reflexión sobre la } \\
\text { práctica, sus competencias, etc. }\end{array}$ \\
\hline De investigación & $\begin{array}{l}\text { - Manifiesta una actitud favorable hacia el aprendizaje continuo y la } \\
\text { innovación. } \\
\text { - Maneja herramientas tecnológicas de aprendizaje. } \\
\text { - Usa documentos bibliográficos. }\end{array}$ \\
\hline Interactivas & $\begin{array}{l}\text { - Empatía con el alumno. } \\
\text { - Promueve la tolerancia, la convivencia, la cooperación, la solidaridad } \\
\text { entre las personas diferentes. }\end{array}$ \\
\hline Éticas & $\begin{array}{l}\text { - Paciente y tolerante con el proceso de aprendizaje del alumno. } \\
\text { - Conoce las características del niño y sus dificultades, aspiraciones, su } \\
\text { entorno social, económico, sus condiciones de vida. } \\
\text { - Asume compromisos y tareas. }\end{array}$ \\
\hline Competencias sociales & $\begin{array}{l}\text { - Establece una relación de confianza con las familias y favorece la } \\
\text { comunicación. } \\
\text { - Colabora con otros agentes educativos. }\end{array}$ \\
\hline
\end{tabular}

Fuente: elaboración propia, basado en Fernández Batanero (2013).

Por tanto, coincidiendo con Fernández Batanero, "el desarrollo de la inclusión requiere un profesorado coherente, es decir, fiel a sus principios y sus creencias. Un profesor que crea en lo que está haciendo y en lo hay que hacer. Un profesor transmisor de valores...” (2OI3).
En el mismo sentido, varios autores son coincidentes con esta idea, Giraldez et al. (2017: $8)$, destacan que, "los mejores materiales, las mejores metodologías, las mejores herramientas pueden ser utilizadas de forma inclusiva o exclusiva. Al final todo depende de que el docente sea un buen profesional y una buena persona”. 
Howard Gardner en una entrevista reciente comentó lo siguiente: una mala persona no llega a ser un buen profesional. A través del experimento Goodwork Proyect, llevado a cabo en Harvard, ha puesto en evidencia que las malas personas no puedan ser profesionales excelentes. Ha comprobado que los mejores profesionales son siempre ECE: excelentes, comprometidos y éticos.

\subsection{Planificación centrada en la persona}

La planificación centrada en la persona (de aquí en adelante PCP) es, ante todo, un enfoque respetuoso, holístico (no reduce a la persona a su discapacidad o trastorno) individualizado y que persigue la capacitación de la persona (Arellano y Peralta, 20I6).

Arellano y Peralta (20I6) consideran la PCP como referencia de prácticas en el ámbito de los servicios socioeducativos, prosigue equiparando dicha filosofía y método de trabajo a la altura de logros como el paradigma de la inclusión, la desinstitucionalización, el modelo de calidad de vida y el movimiento de autodeterminación de las personas con diversidad funcional.

La PCP está emergiendo con fuerza, como medio para responder a las necesidades de las personas, promoviendo su autonomía, su participación y su capacidad para tomar decisiones en áreas significativas de la vida como, por ejemplo, las preferencias educativas y/o profesionales.

Por lo tanto, la PCP aporta nuevas luces para orientar las actuaciones a realizar conjuntamente con las personas con diversidad funcional para facilitar su inclusión social y laboral. La PCP es un proceso de colaboración para ayudar a las personas a acceder a los apoyos y servicios que necesitan para alcanzar una mayor calidad de vida basada en sus propias preferencias y valores. Es un conjunto de estrategias para la planificación de la vida que se centra en las elecciones y la visión de la persona y de su círculo de apoyo (Feaps, 2007).
Todas estas ideas y prácticas conforman la base, los pilares de los que surge la PCP, fundamentada en la importancia del individuo, de sus derechos y, sobre todo, de que es un derecho el que cada uno de ellos pueda controlar y dar forma a su propia vida.

En este sentido, en la preparación para la inclusión laboral desde el escenario educativo, y concretamente desde la secundaria obligatoria y desde las medidas de atención a la diversidad actuales y futuras, se debería trabajar desde este enfoque y conjuntamente con la persona con diversidad funcional: qué es importante para la persona ahora y en el futuro y qué apoyo va a precisar; qué es lo mejor para la persona; qué es práctico y posible para dicha persona.

De este modo, el objetivo de la planificación de los recursos y apoyos socioeducativos ha pasado a ser la persona, apostando por sus capacidades.

Dicho esto, es evidente que una buena práctica que toma como centro a la personas, favoreciendo su inclusión y autodeterminación requiere de un cambio en la manera de plantear la intervención.

Nos referimos con esta idea a que este planteamiento reclama superar enfoques rígidos en el trabajo del docente. Este cambio, que debiera ser inminente, va a depender en gran medida de la implicación y preparación de los profesionales, de sus conocimientos, creencias, percepciones y actitudes.

En definitiva, la implicación de los profesionales es clave.

\section{Conclusiones}

La necesidad de garantizar a todos los estudiantes la participación y el aprendizaje escolar de calidad es hoy en día uno de los mayores desafíos para los sistemas educativos, al igual que lo es favorecer la plena inclusión 
personal, social y laboral de las personas con discapacidad.

Este sistema educativo debe sufrir una profunda transformación para verdaderamente ofrecer a cada alumno una educación de calidad. Con ello nos referimos a que se debe dar un cambio de perspectiva empezando, por ejemplo, por la forma de evaluación y diagnostico de las necesidades educativas del alumnado con discapacidad, para posteriormente centrarse en los puntos fuertes, motivaciones e intereses, olvidando las carencias y limitaciones.

Para ello, se hace necesario un plan basado en destrezas, habilidades y competencias que posibiliten el fomento de la autonomía del alumnado en su aprendizaje.

Por último, aunque no menos importante, está la creación de espacios de colaboración para poder realizar una PCP, la cual entrañe un aprendizaje personalizado, que no necesariamente individualizado, sino organizando y promoviendo metodologías de enseñanza-aprendizaje que desarrollen el aprendizaje participativo, cooperativo y el apoyo entre iguales.

Para terminar, y siguiendo palabras de Pallisera (20II), la PCP, más allá de la transformación de la relación educativa entre personas con discapacidad y profesionales, supone buscar la construcción de relaciones de apoyo mutuo entre las personas. En este sentido, desde una perspectiva social amplia, proponer la construcción de redes de apoyos naturales supone romper las inercias sociales dominantes, avanzando hacia una sociedad basada en relaciones auténticas, de compromiso y solidaridad.

Los profesionales debemos colocarnos las gafas del optimismo, para observar a cada uno de nuestros alumnos con y sin discapacidad como alumnos promesas. 


\section{Referencias bibliográficas}

Arellano, A. y Peralta, F. (2016): "La planificación centrada en la persona: Un ejemplo de buena práctica en el ámbito de la discapacidad intelectual". Contextos Educativos, I9: I952 I 2 .

Echeita, G. et al. (20I7): "Hay que romper con la idea de que todos los niños tienen que aprender lo mismo". Creando Educación para tod@s, I: 9-I 2.

Echeita, G, et al. (2008): La inclusión educativa del alumnado con necesidades educativas especiales, asociadas a discapacidad, en España (en línea). <https://www.uam.es/personal_pdi/ stmaria/sarrio/DOCUMENTOS, $\% 20$ ARTICULOS, $\%$ 2 0 PONENECIAS,/Informe_ final_CIDE_MARZO_o8\% ${ }_{5} \mathrm{~B} 2 \%{ }_{5} \mathrm{D} \%{ }_{5} \mathrm{BI} \%$ ${ }_{5} \mathrm{D} \%{ }_{5} \mathrm{BI} \%{ }_{5} \mathrm{D} \%{ }_{5} \mathrm{BI}_{\mathrm{I}} \%{ }_{5} \mathrm{D} . \mathrm{pdf}>$, acceso $30 \mathrm{de}$ junio de 2008 .

España. Ley Orgánica 8/2013, de 9 de diciembre, para la mejora de la calidad educativa, Boletín Oficial del Estado, ro de diciembre de 2013, núm. 295, pp. 97858-9792I.

Feaps (2007): Planificación Centrada en la Persona. Experiencia de la Fundación San Francisco de Borja para Personas con Discapacidad Intelectual (en línea). <http:// www.acpgerontologia.com/documentacion/ pcpborjasanjuan.pdf $>$, acceso I 8 de diciembre de 2017.
Fernández Batanero, J. M. (20I3): "Competencias docentes y educación inclusiva”. Revista Electrónica de Investigación Educativa, I 5 (2): 82-99.

Giráldez, C. et al. (2017): "La inclusión educativa: de la historia al aula". Creando Educación para tod@s, I: 5-8.

Marchesi, A. et al. (2005): “Assessment of special educational needs integration by the educational community in Spain". European Journal of Special Needs Education, 20 (4): 357-374.

Ministerio de Educación, Cultura y Deporte (2013): Objetivos educativos europeos y españoles. Estrategia Educación Formación 2020 (en línea). <http://www.mecd.gob.es/ $\mathrm{dctm} /$ inee/indicadores $\% \mathrm{E}_{2} \% 80 \%$ 9oeducativos/ informeet 20202013 .pdf? documentId $=0901 \mathrm{e} 72$ b8 $1732 \mathrm{dc} 8>$, acceso I 8 de diciembre de $20 \mathrm{I} 7$.

Pallisera Díaz, M. (20 I I): "La planificación centrada en la persona (PCP): una vía para la construcción de proyectos personalizados con personas con discapacidad intelectual". Revista Iberoamericana de Educación, 56 (3).

Unesco (1994): Declaración de Salamanca y marco de acción para las necesidades educativas especiales (en línea). <http://www.unesco.org/ education/pdf/SALAMA_S.PDF $>$, acceso I 8 de diciembre de 2017. 